\title{
Formación en energías renovables: Resultados obtenidos de experiencias con docentes técnicos de nivel medio.
}

Graciela I. Quiñones ${ }^{1}$; María de los A. Mostajo ${ }^{2}$

\section{RESUMEN:}

E1 trabajo presenta algunos de los resultados obtenidos de la experiencia de formación sobre el tema de las energías renovables de un grupo de docentes de escuelas técnicas de la provincia de Tucumán, en el marco del programa de formación "Profesorado en Educación Secundaria en Modalidad Técnico-profesional en Concurrencia con Título de Base", que coordina el Instituto Nacional de Educación Técnica. El plan de estudio implementado tiene como principal objetivo la formación pedagógico-didáctica, y la actualización científico-tecnológica de los docentes que desarrollan sus actividades en escuelas técnicas de nivel secundario. El módulo Energías Renovables intenta que ellos cuenten con herramientas que le permitan insertar en la escuela la práctica del uso de estas energías no convencionales, y la propuesta de proyectos interdisciplinarios que conduzcan a acciones concretas de mejora en el propio entorno. El análisis y valoración de las posibles acciones arrojó como resultado un listado de planteos cuyas temáticas resultan muy variadas en función de las necesidades, objetivos y recursos de cada comunidad.

PALABRAS CLAVES: educación, energías renovables, eficiencia energética.

\section{ABSTRACT:}

The work presents some of the results of the training experience on the subject of renewable energies, from a group of teachers of technical schools in the province of Tucuman, within the framework of training programs "Teaching staff in secondary education in technical professional modality in concurrence with basic degree" that coordinates the National Institute of Technical Education. The main objective of the implemented study plan is the pedagogical didactic training and the scientific techno-

1 -G. I. QUIÑONES. Centro de Estudios de Energía y Hábitat Sustentable. Facultad de Arquitectura y Urbanismo. Universidad Nacional de Tucumán. Av. Roca 1800.gra_qui@yahoo.com.ar

${ }^{2}$-M. de los A. MOSTAJO. CEEHAS. FAU.UNT. Av. Roca 1800.ttmostajo69@hotmail.com 
logical updating of the teachers who develop their activities in secondary technical schools. The renewable energies module tries that they have tools that allow them to insert in the school the practice of the use of these unconventional energies and the proposal of interdisciplinary projects that lead to some concrete actions. The analysis and assessment of the possible actions resulted in a list of proposals whose themes are very varied according to the needs, objectives and resources of each community.

KEYWORDS: education, renewable energies, energy efficiency.

\section{INTRODUCCIÓN:}

El problema energético actual donde el alza en las tarifas es un hecho, debido entre otras cosas a la disminución de subsidios, se traduce en por ejemplo nuevas políticas energéticas que requiere modificaciones en la formación de los educadores sobre todo de escuelas técnicas para lograr una efectiva transferencia al sector productivo . Para abordar los actuales problemas energéticos se deben desarrollar dos acciones básicas: la sustitución de fuentes energéticas y el uso racional y eficiente.

Por ahora no podemos pensar en prescindir totalmente de las energías convencionales de origen fósil, pero es necesario impulsar el uso de las energías alternativas en los ámbitos donde sea posible hacerlo, para mejorar las tecnologías de aprovechamiento de los recursos renovables y en la producción de energía.

Como sociedad es necesario promover el uso de nuevas fuentes energéticas, aún desde la pequeña escala como puede ser el hogar, y desde la educación, enseñando hábitos de vida

para lograr el adecuado uso de la energía disponible hoy, para preservar las fuentes energéticas en el futuro y lograr cambios globales duraderos.

\section{METODOLOGÍA DE TRABAJO:}

E1 objetivo del módulo Energías Renovables es proveer, de información, fundamentos técnicos y prácticas de utilización sobre el tema de las energías renovables y el uso racional de la energía, también brindar una base de conocimientos que les permita una aproximación a la problemática energética actual y a los campos de acción posibles de abordar para la búsqueda de las soluciones más adecuadas. Transmitirles la importancia de su papel como formadores, ya que serán los responsables de concientizar a sus alumnos sobre el uso de energías renovables como una alternativa para generar energía y mejorar el uso de nuestros recursos naturales mediante la eficiencia energética.

La forma de cursado es presencial y se desarrolla en clases intensivas de tres horas de duración, una vez a la semana.

En la primera clase se evalúan los conocimientos previos de los alumnos sobre los temas del módulo mediante una encuesta con preguntas muy básicas y sencillas de responder, se evalúa a los alumnos sobre el nivel de conocimiento previo del tema, a la vez que permite introducir a los alumnos en la temática de la materia.

En las clases sucesivas se realizan presentaciones en power point con el tema de 
la semana, conceptos principales, ejemplos etc. y para reforzar los contenidos se recurre a la presentación de videos explicativos y la lectura de noticias e informes de actualidad publicados en portales relacionados con la temática, que dan lugar al debate posterior. Luego se desarrollan trabajos prácticos de los temas más relevantes. Estos ejercicios incluyen, entre otros el cálculo de la huella de carbono cuyo objetivo consiste en estimar las emisiones de dióxido de carbono producidas por el uso de energía, el transporte y otras actividades humanas. (Calculador de huella de carbono de la Secretaría de Ambiente y Desarrollo Sustentable de la Nación). Cálculo de superficie necesaria de colectores solares y el volumen del tanque de acumulación de agua para una vivienda familiar. (Instalaciones Complementarias en Edificios de Baja Complejidad), el cálculo de colectores fotovoltaicos para una instalación eléctrica domiciliaria, análisis bioclimático de viviendas, ejercicio donde los alumnos, mediante un croquis de su vivienda, verifican la utilización de las pautas de diseño bioclimático, teniendo en cuenta las condicionantes climáticas del lugar y planteando soluciones constructivas para mejorar el confort de las mismas.

Otra actividad desarrollada fue el " $\mathrm{Ta}$ ller de construcción de un modelo didáctico de horno solar", donde los alumnos pudieron, con materiales muy fáciles de conseguir, verificar el tiempo de cocción de alimentos.
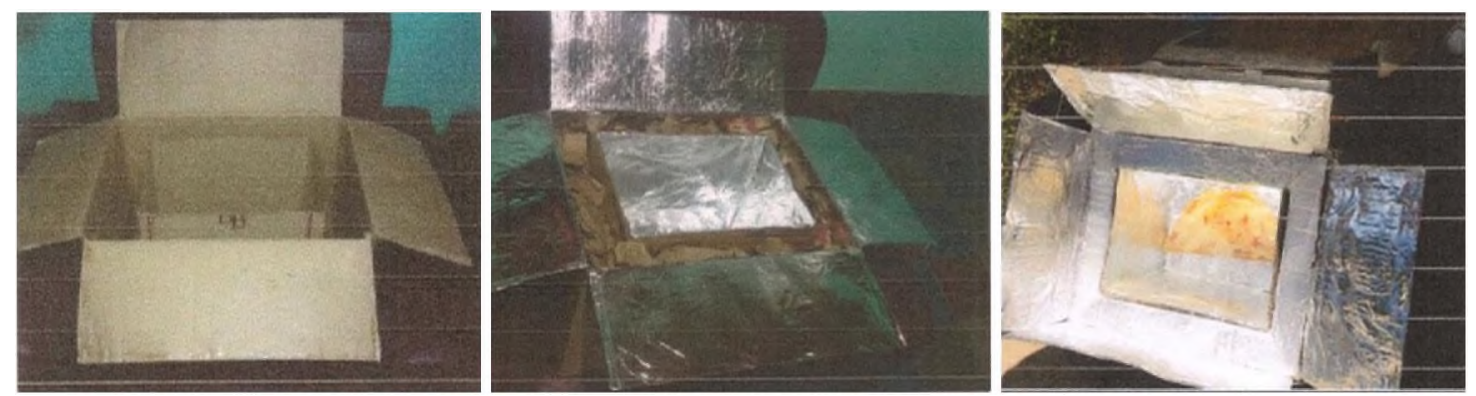

Foto 1, 2 y 3: Armado del horno solar y resultado después de 4 horas de exposición

También se realizó una Exposición de sistemas tecnológicos, pertenecientes al CEEHAS, FAU, UNT, para el aprovechamiento de la energía solar, consistentes en un prototipo de calentador solar para ACS y de un horno solar para la cocción de alimentos. Ambos permiten poner en práctica los contenidos teóricos y vivenciar los resultados de su funcionamiento: el agua caliente en el caso del calentador y la cocción de alimentos en el caso del horno solar.

Por último la evaluación final del curso se planteó con el objetivo fundamental de poner en práctica lo aprendido en el cursado, que posibilite mediante la realización de propuestas de inserción de los temas tratados, en una problemática real 

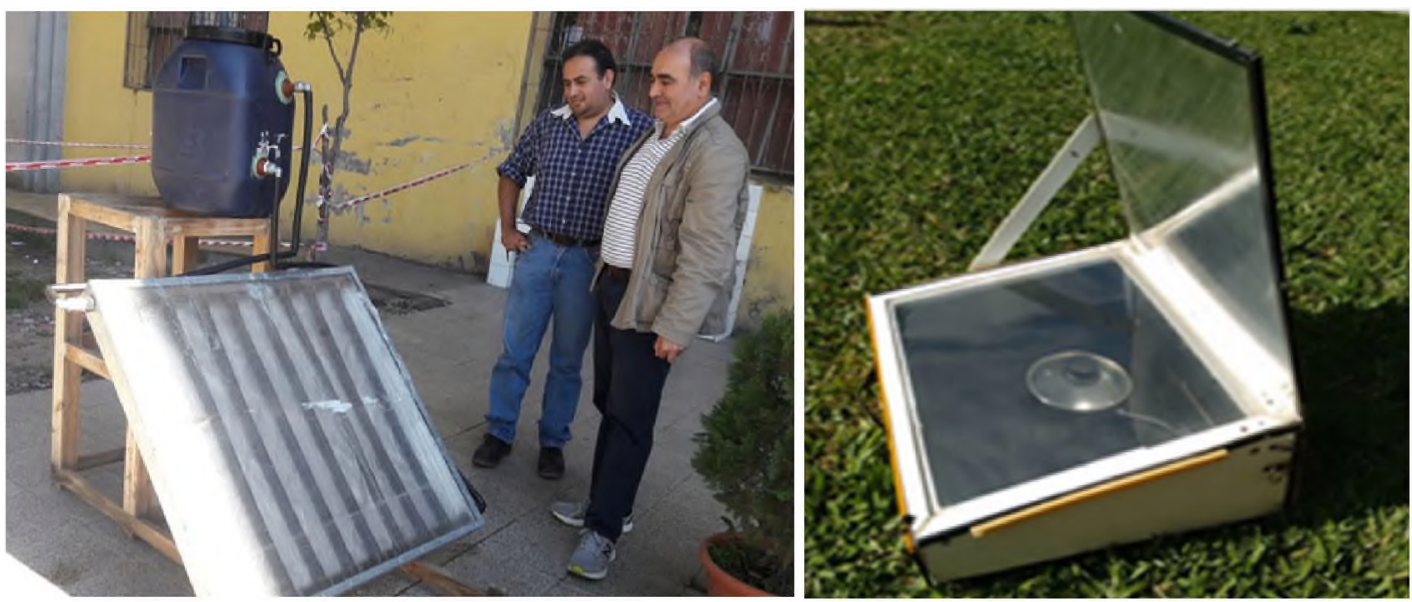

Foto 4 y 5: Exposición de sistemas tecnológicos, colector y cocina solar del CEEHAS

de la comunidad, y tratando de hacer un aportar mínimamente a la solución de la problemática energética global. Consistió en la exposición oral por parte de los alumnos de una propuesta interdisciplinaria de acción que posibilite insertar en la escuela o en su comunidad la práctica del uso de las energías renovables, mediante un proceso participativo de revisión de los planteamientos y prácticas educativas, que conduzca a algunas acciones de mejora en el propio entorno.

Los resultados de las presentaciones abarcaron una gran variedad de temas:

- Diseño de invernadero con botellas Pet y materiales sustentables (prototipo para escuelas Agro técnicas)

- Utilización de la energía solar para el

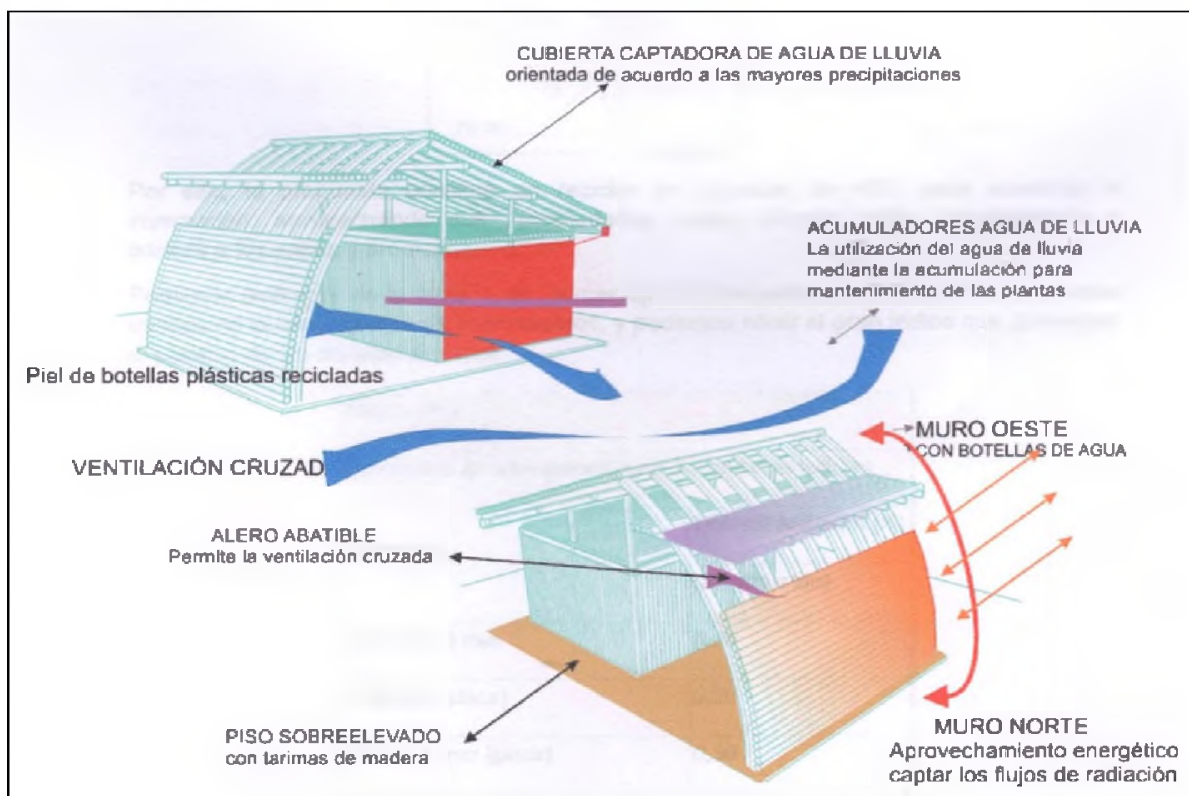

Figura 1: Esquema de comportamiento del invernadero. 
secado de capullos de seda en Esc. Agrotécnica de los Sarmientos, Tucumán.

- Deshidratador solar de frutas y hortalizas para secar la cosecha de la Esc. Agrotecnica de la ciudad de Famailla, Tucumán.

- Instalación de sistema fotovoltaico en la escuela Misión Monotécnica N43, A1derete, Cruz Alta, Tucumán
- Generación de biogás con residuos rurales (Esc. de Agricultura UNT)

- Utilización de techos verdes en sanitario de Esc. Técnica n² 2, Capital, Tucumán

- Aerogenerador, maqueta prototipo para iluminación exterior en escuelas técnicas rurales.

- Obtención de biodiesel a partir del

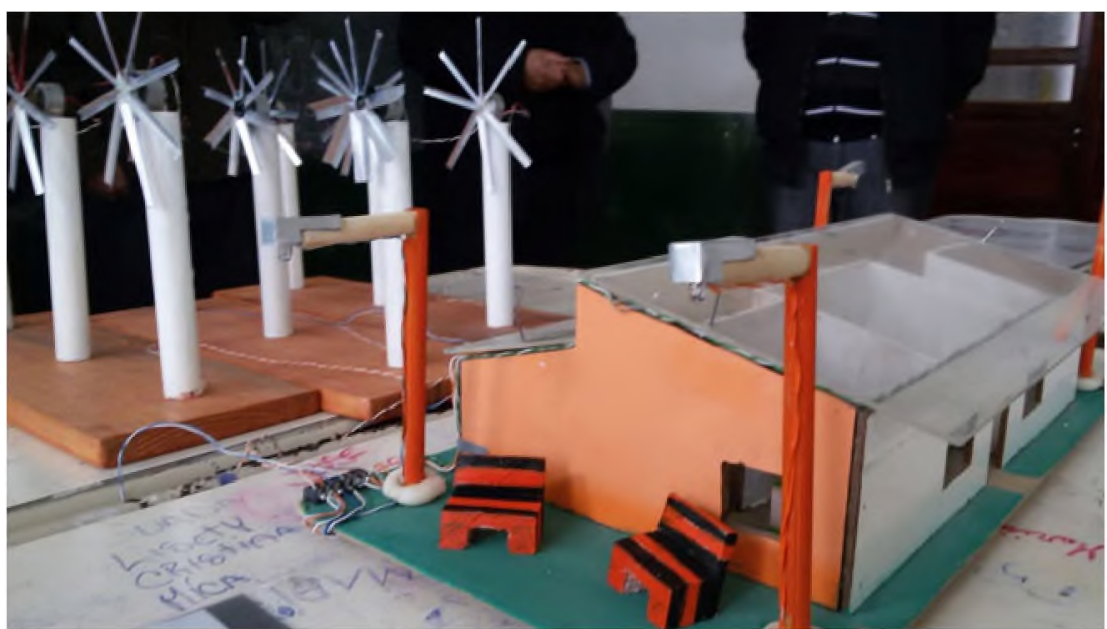

\section{Foto 6: Maqueta de aerogenerador}

uso del aceite vegetal usado (Proyecto para Esc. Técnica de Aguilares, Tucumán

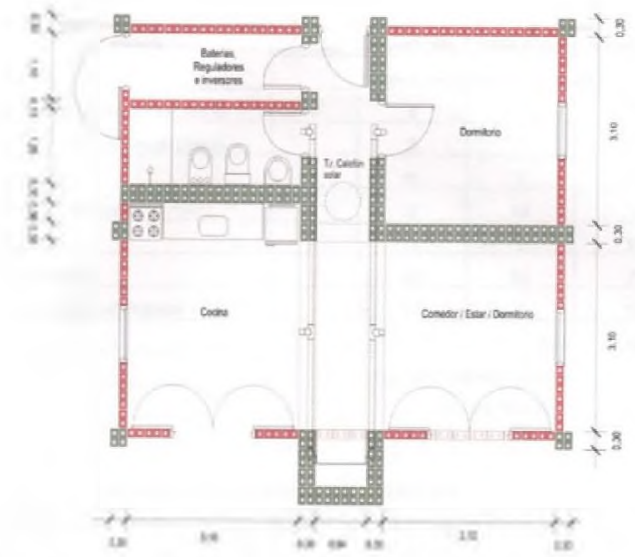

Piane
- Prototipo de vivienda bioclimática de adobe en Trancas, Tucumán

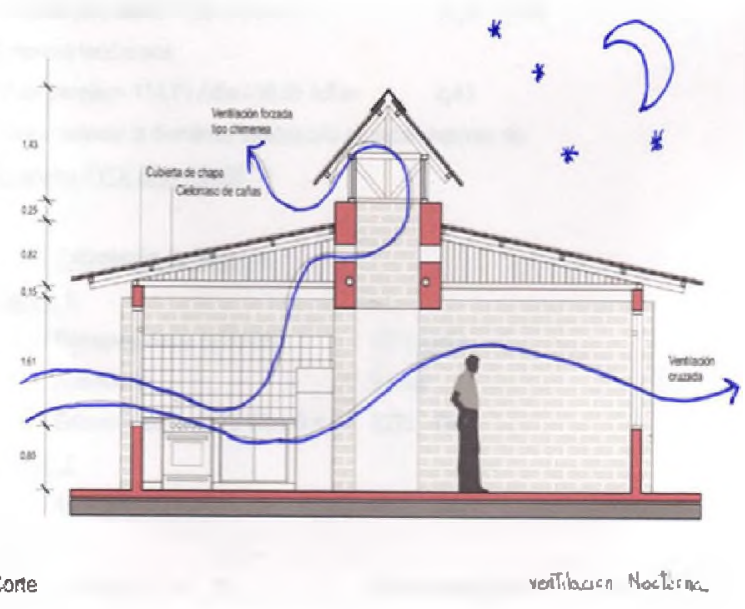

Figura 2 y 3: Planta y corte de comportamiento de la propuesta 

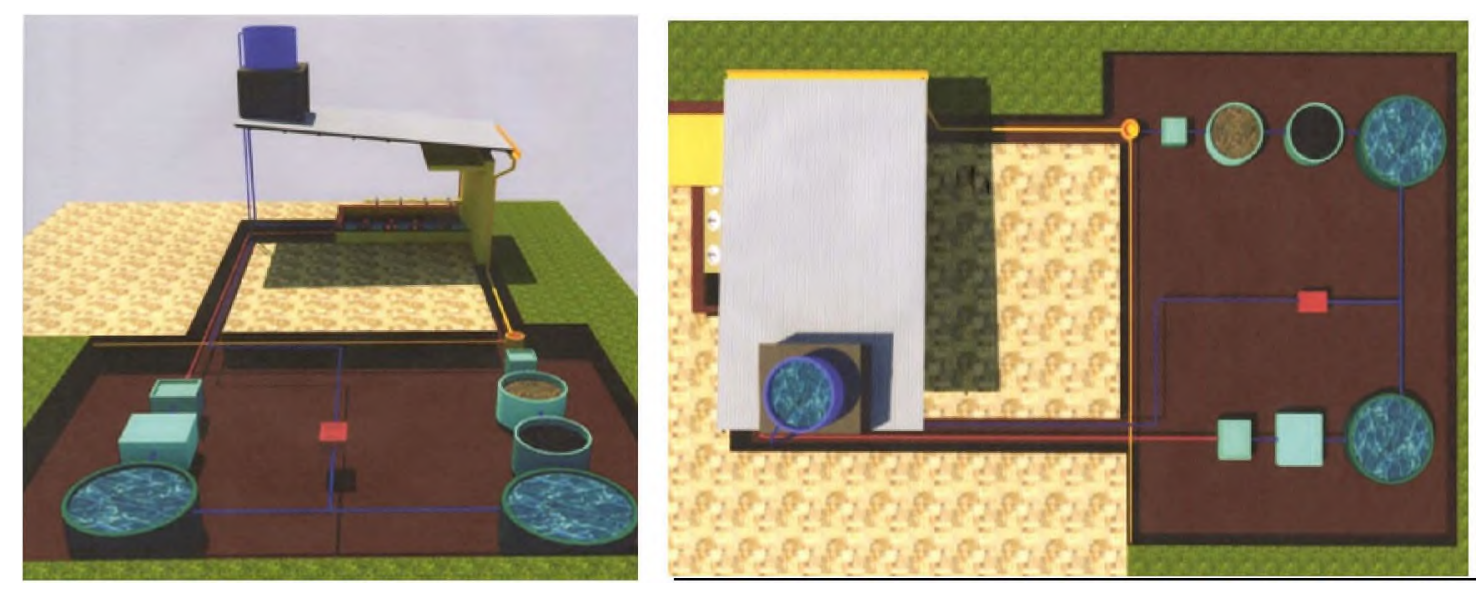

Figura 4 y 5: Maqueta digital del sistema de recuperación de agua

- Proyecto de vivienda bioclimática de adobe con uso de energías no convencionales, Tafi del Valle, Tucumán.

- Recuperación de aguas de lluvia y aguas grises en Esc. Secundaria, Capital, Tucumán.

\section{CONCLUSIONES:}

Las presentaciones finales dejaron ver el importante nivel de interés y participación de los alumnos por la temática en general. Los proyectos presentados fueron muy variados y tenían estudiados todos los aspectos planteados en la consigna del trabajo final como por ejemplo, los objetivos, la interdisciplinariedad, los recursos en general, etc.

En algunos casos profundizaron en los trabajos con modelos demostrativos y con propuestas concretas a desarrollar con alumnos en las escuelas, lo que evidenció la pertinencia y la calidad de las producciones. Les amplió además la perspectiva que tenían sobre los temas estudiados y los movilizó a profundizar en la elaboración de proyectos que den respuesta a problemas específicos de las escuelas y su comunidad.

\section{BILIOGRAFIA :}

- G.E. Gonzalo, 1990 ,Uso racional de la energía y energías no convencionales en la edificación.. Ed. ACFAU. Tucumán.

- E. Mazria , 1983, El libro de la energía solar pasiva.. Ed. G. Gili. México.

- G.E. Gonzalo, 2003 ,Manual de Arquitectura Bioclimática.. 2a edición. Ed. GP 67. Buenos Aires.

- Grabe, Sven, 1987 ,La Educación ambiental en la educación técnica y profesional;; Serie Educación ambiental, UNESCO. Ediciones OREALC. 